\title{
Pallister-Killian syndrome: normal karyotype in prenatal chorionic villi, in postnatal lymphocytes, and in slowly growing epidermal cells, but mosaic tetrasomy $12 \mathrm{p}$ in skin fibroblasts
}

\author{
D Horn, F Majewski, B Hildebrandt, H Körner
}

\begin{abstract}
We report on two patients with PallisterKillian syndrome: an 18 month old male infant followed since the neonatal period and a 4 year old boy. Prenatal diagnosis by chorionic villi sampling (CVS) in the first case showed a normal karyotype without mosaicism. Chromosome analysis on peripheral lymphocytes of the newborn also showed a normal karyotype. The clinical diagnosis of Pallister-Killian syndrome was made after the first year of life because of the typical facial dysmorphism and other characteristic clinical features, such as frontotemporal alopecia, depigmented area of the skin, sensorineural hearing loss, and severe psychomotor retardation. Chromosome analysis from skin fibroblasts now showed an isochromosome 12p mosaicism. The origin of the extra chromosome was confirmed by in situ hybridisation using a chromosome 12 specific library. In the second case chromosomal analysis from peripheral lymphocytes at the age of 19 months showed a normal karyotype 46,XY. Following the clinical diagnosis of PallisterKillian syndrome a superficial skin biopsy was performed which showed very poor and slow growth of cells and a normal karyotype. Because of the typical symptoms a larger and deeper skin biopsy was performed from which there was rapid growth of fibroblasts. Now the diagnosis was established on the basis of the presence of an i(12p) extra chromosome in $69 \%$ of the metaphases.
\end{abstract}

( $($ Med Genet 1995;32:68-71) Medicine (Charite), Luisenstrasse 13 D-10098, Berlin, Germany

D Horn

H Körner

Institute of Human

Genetics and

Anthropology,

Heinrich-Heine

University,

Düsseldorf, Germany

F Majewski

B Hildebrandt

Correspondence to: Dr Horn.

Received 11 April 1994

Revised version accepted for publication 11 August 1994 Pallister-Killian syndrome is caused by tetrasomy $12 \mathrm{p}$ mosaicism. The isochromosome $12 \mathrm{p}$ is rarely found in cultured lymphocytes in the newborn, but mainly in skin fibroblasts or bone marrow cells. In younger children this syndrome is characterised by typical craniofacial dysmorphic features, including frontotemporal alopecia, sparse eyebrows, hypertelorism, flat nasal bridge and a shortened nose, large mouth with downturned corners, severe mental retardation, pigmentary anomalies of the skin, brachydactyly, and the occurrence of diaphragmatic defects and congenital heart defects. ${ }^{1}$ Older children with Pallister-Killian syndrome present a different phenotype with normal distribution of hair, macroglossia, prognathism, seizures, and profound mental retardation. ${ }^{2}$ Prenatal diagnosis of tetrasomy $12 p$ by CVS and amniocentesis has been documented in several cases. ${ }^{3-8}$ The isochromosome $12 \mathrm{p}$ in amniotic cells and chorionic cells in most of these cases was observed in all or nearly all cells.

\section{Case reports}

CASE 1 (BERLIN)

Prenatal diagnosis by CVS, requested by a 45 year old German mother and her 48 year old German husband, showed a normal karyotype, $46, X Y$. The family history was unremarkable. Pregnancy was complicated by polyhydramnios. Spontaneous delivery occurred in the 36th week of pregnancy. Birth weight was $2870 \mathrm{~g}$, length $49 \mathrm{~cm}$, and OFC $36 \mathrm{~cm}$. Dysmorphic features noted were a prominent metopic suture, short palpebral fissures, a short nose with depressed bridge and anteverted nares, simple and elongated philtrum, a large mouth, bifid uvula, receding chin, and dorsally rotated ears (fig 1A, B). A short neck with excessive nuchal skin, widely spaced nipples, and undescended testes were noted. The upper and lower limbs and the hands and feet were short. Ulnar deviation of all fingers (fig 1C) and simian creases were observed as well as a ventricular septal defect. Radiological examination showed brachydactyly and dislocation of the heads of the radii. Chromosome analysis on peripheral lymphocytes showed a normal karyotype, $46, \mathrm{XY}$.

At 10 months of age the boy was $71 \mathrm{~cm}$ long a 10 of $43 \mathrm{~cm}(<3 \mathrm{rd}$ ( occiput and face were now flat. Temporofrontal alopecia, depigmentation over the right eyebrow, a shallow upper orbital ridge, hypertelorism, and ptosis were obvious. The mouth was large with downturned corners (fig 2A, B). No teeth had emerged. Ophthalmological examination showed nystagmus and exophoria. The ulnar deviation of the fingers with the exception of the index finger had resolved. Profound motor and mental re- 


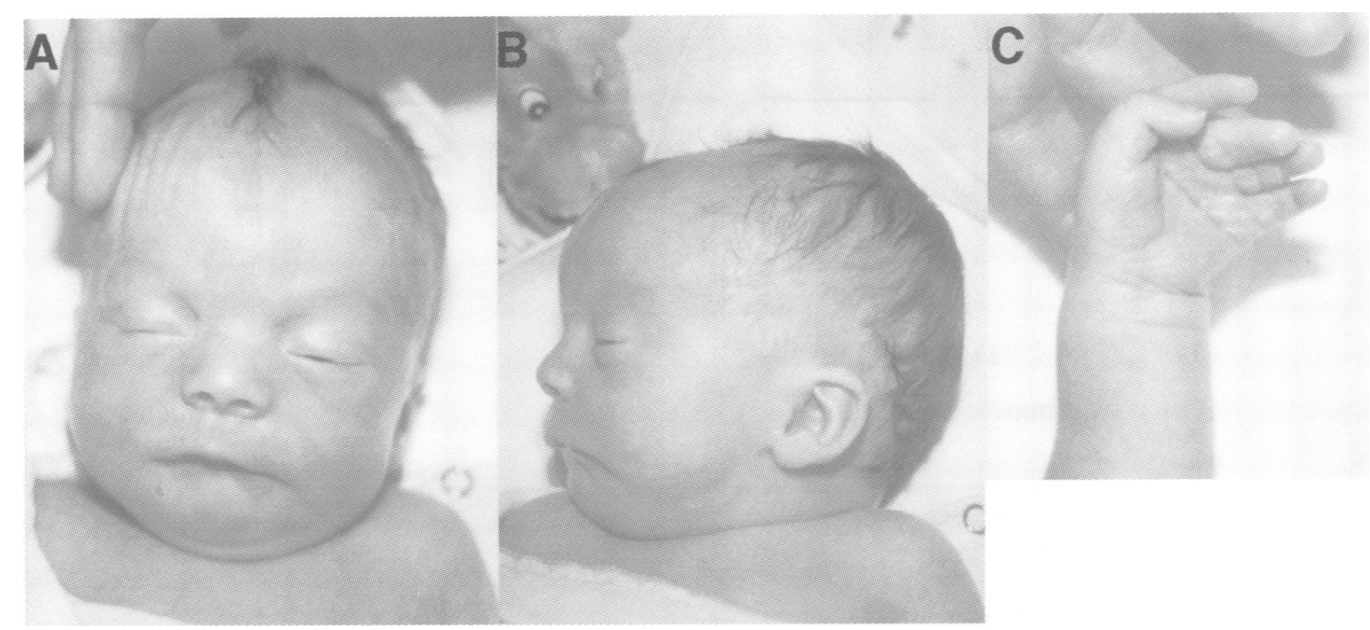

Figure 1 Case 1 as newborn showing $(A)$ short palpebral fissures, short nose, simple philtrum, large mouth, short neck, (B) small mandible, low set, dysplastic ears, alopecia of the forehead, (C) ulnar deviation of all fingers and simian crease.

tardation, generalised muscular hypotonia, and sensorineural hearing loss were noted. Cerebral CT scan showed slight dilatation of the ventricles.

\section{CYTOGENETIC INVESTIGATIONS}

Seventeen cells from the direct preparation and eight cells from cultured chorionic villi were examined. $\mathrm{G}$ banded chromosomes had a normal male karyotype. Additionally, a retrospective investigation of 28 mitoses of the chorionic cell samples showed no evidence of chromosomally abnormal cells. The striking phenotype of the newborn led us to perform a cytogenetic investigation of peripheral blood lymphocytes. $\mathrm{G}$ banded chromosome studies of 100 mitoses showed a normal karyotype. At the age of 18 months a skin biopsy was performed and was cultured in Amniomax medium. Karyotyping of fibroblasts showed the presence of an isochromosome $12 p$ in $85 \%$ of 100 mitoses (fig 3A). The origin of the extra chromosome was confirmed by in situ hybridisation using a chromosome 12 specific library (fig 3B).

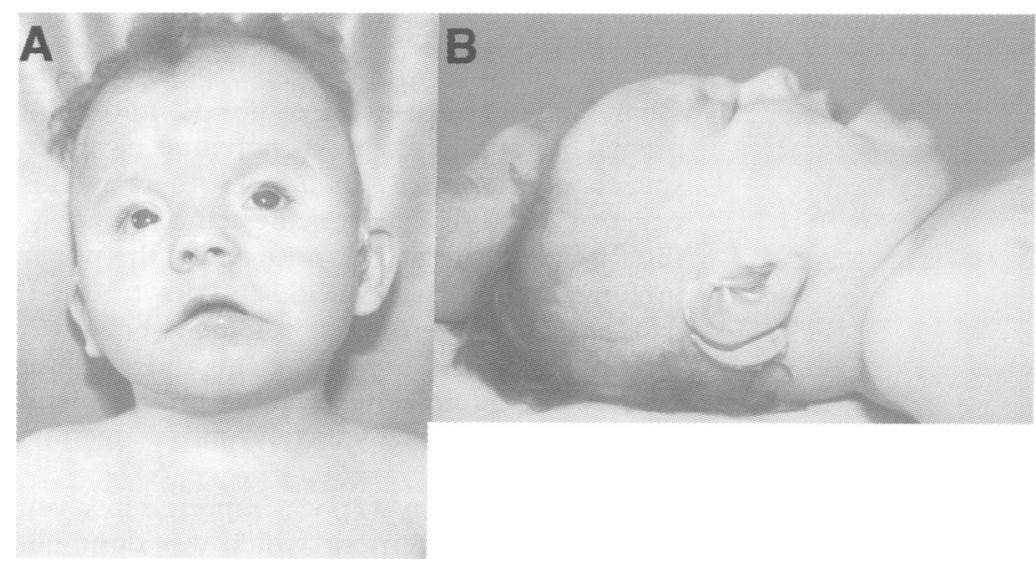

Figure 2 Case 1 at the age of 1 year showing $(A)$ high frontal hairline, prominent metopic suture, depigmentation over the right eyebrow, ptosis, hypertelorism, short nose with upturned nares, large mouth with downturned corners, and (B) temporofrontal alopecia and flat occiput.
CASE 2 (DÜSSELDORF)

This was the third child of healthy, unrelated parents. Spontaneous birth occurred at 38 weeks of gestation. Birth weight was $4220 \mathrm{~g}$, length $54 \mathrm{~cm}$, and OFC $37 \mathrm{~cm}$. The boy had alopecia of the forehead, reduced spontaneous movements, and retarded motor and speech development. He sat at 3.5 years. At the age of 4 years he could not speak, was unable to stand, and had no contact with his parents or other persons. We saw the boy first at the age of 19 months, and noted a prominent glabella, marked alopecia of the forehead, and small depigmented areas in this region, a shortened nose, long upper lip, broad mouth, multiple frenula at the upper alveolar ridge, large and deep set ears, loose skin with three further depigmented spots, muscular hypotonia, no fixation, and marked developmental delay.

Chromosome analysis on lymphocytes showed a normal male karyotype and we made the tentative diagosis of Opitz trigonocephaly syndrome. When looking at the slides 20 months later, we corrected our diagnosis to Pallister-Killian syndrome. At re-examination at the age of 3 years 4 months the boy was still severely retarded, with no further motor or speech development. His length was $99 \mathrm{~cm}$, his weight $17 \mathrm{~kg}$, and his OFC $52 \mathrm{~cm}$ (fig 4). We informed the parents of our diagnosis, but chromosomal analysis from epidermal cells failed to show the expected extra chromosome. Since growth of the epidermal cells was very slow and poor and the facial aspect and the degree of mental retardation corresponded closely to the Pallister-Killian syndrome, we performed a second skin biopsy when the patient was 4 years old. Since this skin biopsy was deeper than the first one, there was fast and ample growth of fibroblasts.

At the age of 4 years, height $(102.5 \mathrm{~cm})$, weight $(17.5 \mathrm{~kg})$, and $\mathrm{OFC}(52.5 \mathrm{~cm})$ were normal. The scalp hair was now thick and curly, the frontal alopecia was less striking than at the first examination, the nose was short, the philtrum long, the mouth wide, and the ears large. The enlarged tongue protruded and prognathism developed which becomes more pronounced in adult patients. ${ }^{2}$ 


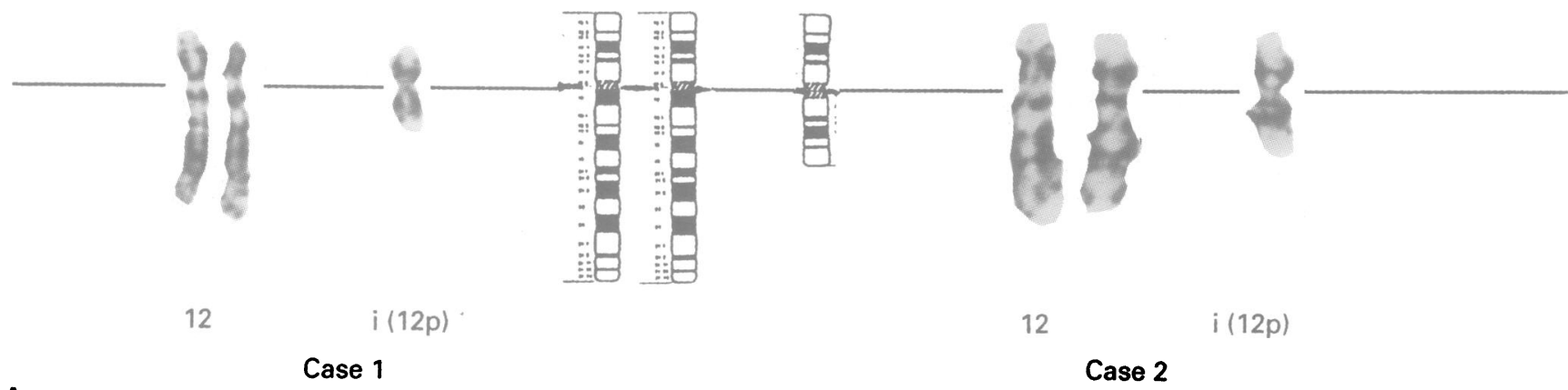

A

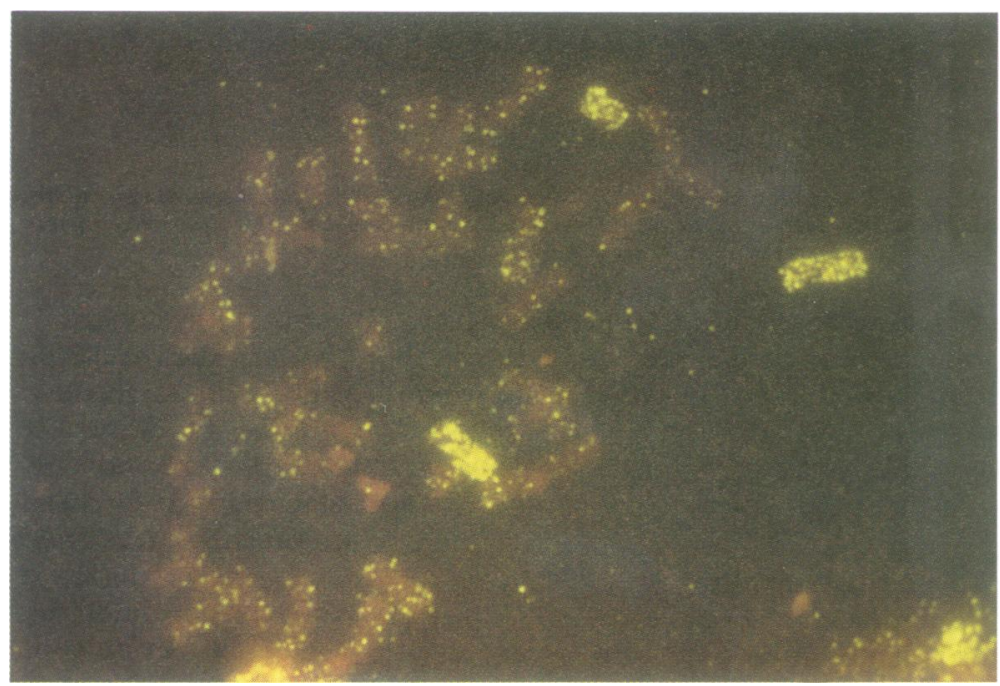

Figure 3 Case 1. (A) G banded and graphic partial karyotype of case 1 and case 2 from skin fibroblasts. (B) Note isochromosome 12p. In situ hybridisation with chromosome 12 specific library showed the presence of two normal chromosomes 12 and the isochromosome $12 p$.

CYTOGENETIC INVESTIGATIONS

Chromosome analysis from peripheral lymphocytes showed a normal karyotype $46, \mathrm{XY}$ at the age of 19 months. At the age of 3 years 4 months a superficial skin biopsy was performed and was cultured in Chang-D medium. There was a very slow growth of epidermal cells; the first harvest was done after six weeks and the second harvest after seven weeks. Fifty me- taphases were analysed, and none showed the expected extra chromosome. At the age of 4 years, a second skin biopsy was performed to a greater depth $(2 \mathrm{~mm}$ in depth and 4 to $5 \mathrm{~mm}$ in diameter) and now there was a rapid growth of fibroblasts using the same cultural procedure; after the first passage we now observed an $i(12 p)$ extra chromosome in $69 \%$ of the metaphases (31/45) (fig 3A).

\section{Discussion}

The Pallister-Killian syndrome is caused by a tissue specific mosaic i(12p) extra chromosome mainly in skin fibroblasts or bone marrow cells, and only rarely in lymphocytes of the newborn. Characteristic features are profound motor and mental retardation; most patients have no speech development. In infancy there is alopecia of the forehead and the temporal areas, the forehead is high, the nose short, the upper lip long, and the mouth wide and downturned. The chin is receding. The tongue is enlarged, causing a protruding lower lip and in later infancy and adolescence marked prognathism. There may be brachydactyly and congenital heart defects.

Mosaic tetrasomy $12 \mathrm{p}$ was reported in seven cases after amniocentesis which was done because of advanced maternal age or ultrasound abnormalities. ${ }^{35-7}$

A high proportion of cells with the isochromosome $12 \mathrm{p}$, which is consistent with the
Figure 4 Appearance of case 2 at the age of 3 years 4 months: high forehead with frontotemporal alopecia, short nose, enlarged tongue, broad mouth, and large ears. 
results in postnatal skin fibroblasts, was observed in all cases. Prenatal diagnoses of mosaic tetrasomy $12 p$ by CVS were reported by Sharland et $a l^{4}$ and Bresson $e t a l .{ }^{8}$ In both cases a similarly high percentage of tetrasomic cells was detected. In our case 1 with PallisterKillian syndrome a normal karyotype in direct preparation and in long term culture of chorionic tissue was observed. The advanced maternal age suggests the possibility that the aneuploidy observed later in the patient resulted from a meiotic error with subsequent mitotic loss of the isochromosome in some cells during embryogenesis. The normal karyotype of the cytotrophoblast (direct chromosome preparation or short term incubation of the villi) and of the mesenchymal core of the villi (long term culture) could be because of very early loss of the extra chromosome in some cells or the fact that only a few chorionic cell clones could be examined.

The non-disjunction and the isochromosome formation may occur postzygotically after the differentiation between cytotrophoblast, mesenchymal core, and the embryo; this could explain the normal chromosome analysis in chorionic villi.

The dysmorphism of the face suggestive of a chromosome aberration led us to perform a cytogenetic investigation from peripheral lymphocytes with normal results in both cases.

In the reported newborn cases with the isochromosome $12 p$ the incidence of aberrant metaphases in blood cultures is very low. ${ }^{19}$ Results in fetal lymphocytes are very different; the percentage of cells with the extra chromosome was $1 \%, 3 \%, 5 \%$, and $100 \% .^{35-7}$

Clinical diagnosis and investigation of skin fibroblasts with the presence of $85 \%$ tetrasomic cells were done after the proband's first year in case 1. Aneuploid cells in cultured fibroblasts are initially present in a high percentage. After several in vitro passages of cultured skin fibroblasts or other tissue cells, the isochromosome can be lost, as is shown by our case 2 . The same observation has been made by some authors. ${ }^{9-11}$ It is possible that $\mathrm{i}(12 \mathrm{p})$ fibroblasts have a slower growth than diploid cells.

In cases with the typical symptoms of Pallister-Killian syndrome skin biopsy should be repeated if cell growth is poor in the first cultures. No correlation between the proportion of tetrasomic cells in different tissues investigated and the severity of the condition is known so far.

We would like to thank Mrs Karin Lehmann for in situ hybridisation studies.

1 Schinzel A: Tetrasomy 12p (Pallister-Killian syndrome). $\mathcal{f}$ Med Genet 1991;28:122-5.

2 Horneff G, Majewski F, Hildebrand B, Voit T, Lenard HG Pallister-Killian syndrome in older children and adolescents. Pediatr Neurol 1993;9:213-5.

3 Gilgenkrantz S, Droulle P, Schweitzer M, et al. Mosaic tetrasomy 12p. Clin Genet 1985;28:495-502.

4 Sharland M, Hill L, Patel R, Patton M. Pallister-Killian syndrome diagnosed by chorionic villus sampling. Prenot Diagn 1991;11:477-9.

5 Shivashankar L, Whitney E, Colmorgen G, et al. Prenata diagnosis of tetrasomy $47, \mathrm{XY}, \mathrm{i}(12 \mathrm{p})$ confirmed by in situ hybridization. Prenat Diagn 1988;8:85-91.

6 Soukup S, Neidich K. Prenatal diagnosis of Pallister-Killian syndrome. Am 7 Med Genet 1990;35:526-8.

7 Tejada MI, Uribarren A, Briones P, Vilaseca MA. A further prenatal diagnosis of mosaic tetrasomy 12p (PallisterKillian syndrome). Prenat Diagn 1992;12:529-34.

8 Bresson JL, Arbez-Gindre F, Peltie J, Gouget A. PallisterKillian mosaic tetrasomy $12 \mathrm{p}$ syndrome. Another prenatally diagnosed case. Prenat Diagn 1991;11:271-5.

9 Wenger SL, Steele MW, Yu W-D. Risk effect of maternal age in Pallister $\mathrm{i}(12 \mathrm{p})$ syndrome. Clin Genet 1988;34: age in

10 Peltomäki P, Knuutila S, Ritvanen A, Kaitila I, de la Chapelle A. Pallister-Killian syndrome: cytogenetic and molecular A. Pallister-Killian syndrome: cytogen

11 Thornburg Reeser SL, Wenger SL. Failure of PHA-stimulated $\mathrm{i}(12 \mathrm{p})$ lymphocytes to divide in Pallister-Killian syndrome. Am $\mathcal{Y}$ Med Genet 1992;42:815-9. 